\title{
A Parametric Study of Binary Sequence
}

\author{
Youssef Khmou \\ Sultan Moulay Slimane University, Morocco \\ khmou.y@gmail.com
}

\begin{abstract}
We study, in this paper, several properties of the binary sequence defined by the values $(0,1)$, we present a review of the high order statistics as function of the average value known as the density. Different entropy functions, applied in information theory and statistical mechanics, are presented where their expressions are derived as function of the density. In the second part, we partially study the randomness characteristic of the binary sequence, based on a recently proposed metric inspired from diffraction theory, we present a new function whose expression is based on a principal equation in the field of antennas and propagation, based on the array factor which gives the interference pattern of uniform linear array of antennas in the far field region, we propose a real valued function in order to characterize the geometric repartition of the binary values of the sequence. Numerical simulations show its consistency with Shannon entropy. For binary sequence generated from logarithmic logistic map, the obtained results indicate the equivalency between this function and the diffraction based metric.
\end{abstract}

Keywords: binary sequence, statistics, entropy, randomness

\section{Introduction}

A binary sequence $[1,2,3,4]$ is vector $x$ defined by its number of elements or equivalently its length $L$ such that each element $x_{n}$ for $n=1, \ldots, L$ can be equal to one of two possible measurable values, the sequence can refer to measurements or simulated data with respect to the space or time dimension. In computer science [5], the data are treated in binary form which consists of strings of zeros and ones. In electronics [6] and communication sciences $[7,8,9]$, a binary sequence consists of signal where at given time, the amplitude of signal equals one of two values, several kinds of the signal are possible including the configuration $\{v, 0\}$ where $v>0$ is the voltage or current for example, the configuration of $\{-v, 0\}$ or the symmetrical signal $\{-v,+v\}$. Another configuration consists of two symbols for example tow letters 'A' and 'B' such that the Fibonacci and Thue-Morse sequences [10]. All these types can be transformed into binary sequence with values $(1,0)$ due to its simplicity for parametric analysis. The applications of the binary sequences span several fields including for example biology [11], information science [12], economical and physical [10] sciences. In computational physics [5], the binary sequence is model of the one dimensional Ising model that describes a system in thermal equilibrium, each particle of the system is modeled by its spin which can take one of two values $s= \pm 1 / 2$ and the particles are described by a state vector $s=\left[s_{1}, s_{2}, \ldots, s_{L}\right]$ which can take $2^{L}$ different states for $L$ particles.

In the field of computation traffic flows $[13,14]$, the binary sequence is also very important concept for modeling the traffic based on cellular automata models, considering

Received (May 12, 2018), Review Result (July 21, 2018), Accepted (July 31, 2018) 
for the example a section of road sampled into $L$ sites, each site can either be occupied by single vehicle or empty, a configuration of the traffic based on its input parameters for simulation forms logical array of zeros and ones. The properties of the traffic are derived from the binary matrix including for example the spatial and temporal densities.

Focusing on the type of sequence $x$ with values $(1,0)$, the occurrence probability of the value 1 is simply the average value, that is the sum of the elements $x_{n}$ divided by the length, another important property that characterizes the sequence is the geometrical configuration which is the repartition pattern of the binary values of $x$. If the number of the values 1 is fixed, it is obvious that the probability density function is also fixed for any repartition, however the spectral and other statistical properties depend on of the pattern of repartition including for example the correlation properties [15]. The repartition pattern can for example be periodic or random. The randomness property of $x$ is analyzed using correlation functions. Indeed, using the spectral modification of $x$, the authors in [16], proposed a method for generating correlated random binary sequences based on iterative filtering algorithm. The output of the iterative filter yields a sequence whose correlation pattern can be exponential for example, or can be characterized by spectrum of colored noise.

Given two random sequences, comparing which one is more random than the other may require additional metrics to quantify the degree of randomness. In recent study [17], the authors proposed a complex valued function, inspired from the diffraction theory [18], in order to measure the disorder of binary sequence, the authors found that the variation of their metric is statistically equivalent to several entropy functions, the numerical tests were based on different methods such as periodic binary sequences and the logistic map [5]. In the same context we present a variant of this function, in order to partially study the randomness behavior of binary sequence $x$, we propose a real valued function whose expression is based on an important equation in the field of antennas and propagation [19], the expression of our function is based on the concept of the array factor which is a function that describes the interference pattern of an ensemble of identical and uniformly placed antennas, in the far field region from the array, we compare its variation with the proposed complex valued function using several computer simulations.

This paper is organized as follows, in the second part, we briefly present different statistical properties of a binary sequence $x$, all the presented statistical metrics are expressed as a function of the density. Next, we present simplified expressions of several entropy functions applied in the fields of information theory and statistical mechanics. In the third part, we partially treat the problem of the randomness of the binary sequence, by proposing a variant of a recently proposed function, and we compare their variation where we illustrate and discuss the obtained numerical results.

\section{Statistical Properties of Binary Sequence}

A binary sequence is one dimensional vector $x$ where each element can take one of the two possible values, for example in the field of telecommunications [6], the symmetric binary signal consists of two values $+v$ and $-v$ where $v$ is constant. In non linear science and computational physics, a binary sequence can be considered as one dimensional cellular automaton model where each site can either be occupied or empty, thus the two possible values are 1 and 0 forming a logical array, an example of such model is in traffic flow modeling using cellular automata where each site can either be occupied by one vehicle or empty. Based on these definitions, we analyze theoretically and numerically some properties of binary sequence, we consider a vector $x$ of length $L$ where each element is equal to 1 or 0 , the vector is given by the sequence $x=\left[x_{1}, x_{2}, \ldots, x_{L}\right]$. The first fundamental property of $x$ is the 
occupation probability $p(1)$, which is the number of the components that are equal to1. Complementarily, the other probability is $p(0)=1-p(1)$. Instead of using the expression of the occupation probability, we consider the definition of the density $\rho$ which is, for example in traffic flow modeling, the number of vehicles per length $L$, thus we have the following relation:

$$
\rho=<x>=p(1)=\frac{N}{L}=\frac{1}{L} \sum_{n=1}^{L} x_{n}
$$

Where $N$ is the number of occupied sites or the number of elements that are equal to 1 with the condition $0 \leq N \leq L$. The first remark that we can deduce from the definition of $\rho$ is the equilibrium between empty and occupied sites, when half of the elements equal 1 and the other half equal 0 we obtain a critical density $\rho_{c}$ which is computed using the following relation:

$$
\rho_{c}=\frac{1}{L(L+1)} \sum_{N=0}^{L} N=\frac{1}{2}
$$

Note that this value can also be computed if we consider $\rho$ as continuous variable with boundary values $\rho_{\min }$ and $\rho_{\max }$, thus we obtain the following definition:

$$
\rho_{c}=\frac{1}{\rho_{\max }-\rho_{\min }} \int_{\rho_{\min }}^{\rho_{\max }} \rho d \rho=\frac{\rho_{\max }+\rho_{\min }}{2}
$$

If we have set the boundary values $\rho_{\min }=0$ and $\rho_{\max }=1$, then the critical density is similarly to the discrete case equal to $\rho_{c}=0.5$. The variable $\rho$ is the first order statistic of the sequence $x$, high order statistics can also be expressed in terms of $\rho$. The relation of the standard deviation is given by:

$$
\Delta \rho=\sqrt{<(x-\rho)^{2}>}=\sqrt{\rho-\rho^{2}}
$$

Where the operator $\langle f\rangle=(1 / L) \sum_{n=1}^{L} f_{n}$ denotes the ensemble average, the expression of the standard deviation is based on the property $\left\langle x^{2}\right\rangle=\langle x\rangle=\rho$ since we have $x_{n}^{2}=x_{n}$. Next, the variance is given by $\operatorname{var}(x)=(\Delta \rho)^{2}=\rho-\rho^{2}$. The variance is maximal at critical density $\rho_{c}=1 / 2$ and equals $\operatorname{var}(x)=1 / 2$. Among the possible interpretations of the variance is in traffic flow modeling, based on Greenshields model [20] where the velocity decreases linearly with increasing density, the fundamental diagram is constructed using the relations of the speed and the flow as:

$\left\{\begin{array}{l}v=\alpha-\beta \rho \\ J=v \rho=\alpha \rho-\beta \rho^{2}\end{array}\right.$

Where $\alpha$ and $\beta$ are constants that can be estimated using real data. If we consider a normalized version of the above model where we set $\alpha=\beta=1$ with jam density $\rho_{\max }=1$, we obtain simplified version of the flow-density relation which 
can be expressed in terms of the variance of the binary sequence $x$, given the speed $v=1-\rho$, the flow as a function of the variance is given by:

$J=p(1) p(0)=\rho(1-\rho)=\operatorname{var}(x)$

Another interesting metric is called variance coefficient, it is defined by the standard deviation over the average value and is used in the field of information theory, the variance coefficient denoted by letter $c$ is given by:

$c=\frac{\Delta \rho}{\rho}=\sqrt{\frac{1-\rho}{\rho}}$

Given the probability density function or the normalized histogram of random variable, the skewness is a function that quantifies the asymmetry of the probability density function with respect to the average value. In our case, the expression of the skewness is reduced into the following equation:

$s=\left\langle\left(\frac{x-\rho}{\Delta \rho}\right)^{3}\right\rangle=\frac{1}{\Delta \rho^{3}}<x^{3}-3 x^{2} \rho+3 x \rho^{2}-\rho^{3}>=\frac{1}{\Delta \rho^{3}}\left(2 \rho^{3}-3 \rho^{2}+\rho\right)$

We remark from the above that if the symbols of the binary sequence have the same probability, such that the density is $\rho=0.5$ for binary values $(1,0)$, the histogram is symmetric which implies that the skewness equals zero. The kurtosis $k$ is descriptive metric of the shape of the probability density function, for binary sequence, its expression is reduced into the following equation:

$$
k=\left\langle\left(\frac{x-\rho}{\Delta \rho}\right)^{4}\right\rangle=\frac{1}{\Delta \rho^{4}}\left(-3 \rho^{4}+6 \rho^{3}-4 \rho^{2}+\rho\right)
$$

Similarly, the expressions of the high order moments can have derived as function of the density. The statistical properties of the binary sequence $x$ can also be studied using the formalism of the information theory, precisely the entropy functions. Given the sequence with values $(1,0)$, the variations of the entropy functions indicate that the maximal values correspond to the average density $\rho=0.5$. Among the well known functions is the Shannon entropy [21] defined by the equation $h(\rho)=-\rho \log (\rho)-(1-\rho) \log (1-\rho)$ where the maximal value is $\log (2)$. We briefly present some other examples of the entropy functions based on the information theory and statistical mechanics. The Rény entropy [22] is defined by the set of probabilities $p_{1}, \ldots, p_{N}$ with parameter $q$ such that $0<q<+\infty$ and $q \neq 1$, it is given by the equation:

$$
h(p, q)=\frac{1}{1-q} \log \left(\sum_{i=1}^{N} p_{i}^{q}\right)
$$

For special value of the parameter $q=2, h(p, 2)$ is called collision entropy where it is applied in the field of quantum information theory [22]. For our case of binary sequence, the collision entropy can be derived as the following:

$$
h(\rho, 2)=-\log \left(2 \rho^{2}-2 \rho+1\right)
$$


The maximal value corresponds to the average density $\rho=0.5$ and equals $h(0.5,2)=\log (2)$ similarly to the Shannon entropy. In statistical mechanics, The Tsallis entropy [23] is a generalization of the Boltzmann-Gibbs entropy that describes classical thermodynamical system with microstates given by the probabilities $p_{i}$ for $i=1, \ldots, \Omega$. It is defined by parameter $\alpha$ as:

$$
h(p, \alpha)=\frac{k}{\alpha-1}\left(1-\sum_{i=1}^{\Omega} p_{i}^{\alpha}\right)
$$

Where $\alpha>0$ and $\alpha \neq 1, k$ is the Boltzmann constant which we consider in this framework as $k=1$. Let us consider that the parameter $\alpha=2$, using the states of the binary sequence, the corresponding Tsallis entropy is given by the following equation:

$h(\rho, 2)=2\left(\rho-\rho^{2}\right)$

Comparing the above expression with that of the variance, we remark that Tsallis entropy with $\alpha=2$ is proportional to the variance function, of the binary sequence, described previously. Considering the Boltzmann-Gibbs entropy, if all the microstates have the same probability $p_{i}=1 / \Omega$, the Boltzmann entropy [24] is given by its usual expression:

$$
h=k \log (\Omega)
$$

For binary sequence $x$, we apply the Boltzmann entropy based on the concept of cellular automaton model. We consider the traffic flow of particles or vehicles with periodic boundary conditions. Given a lattice of length $L$, we analyze from theoretical viewpoint, the geometric configuration of the vehicles given fixed density $\rho$. Each site of the lattice can either be occupied by single vehicle or empty, the allowed values of the system are $(1,0)$. Similarly to statistical mechanics where $N$ particles can occupy $L$ levels of energy, we consider that $N$ vehicles can be distributed in $L$ sites, the number of the microstates in this case equals $C_{L}^{N}$ on the condition that the particles are indiscernible with boundary conditions $C_{L}^{0}=C_{L}^{L}=1$ based on the convention $0 !=1$. Projecting this concept on binary sequence $x$ of length $L$, given $N$ values of 1 and $L-N$ values of 0 , the number of the microstates is also equal to $\Omega=C_{L}^{N}$. For example a lattice of $L=100$ sites, the maximum value of $\Omega$ reaches $10^{28}$ if $N=L / 2$, to reduce this value and to obtain correct boundary conditions for $N=0$ and $N=L$, we use the logarithm of $\Omega$, therefore we obtain the Boltzmann entropy of the binary sequence $x$ as the following:

$$
h=k \log (\Omega)=k \log \left(C_{L}^{N}\right)=k \log \left(\frac{L !}{(L-N) ! N !}\right)
$$

The variation of $h$ can be defined by the index of the maximal value and the values of the boundary conditions, $h(N=0)=h(N=L)=0$ and the maximum of $h$ corresponds to $N=L / 2$ which is equivalent to the critical density $\rho_{c}=0.5$. From this comparative review of the different entropy functions, we remark that theirs variations are similar with respect to the density, such that any given entropy 
metric $h$ as function of $\rho, h$ separates two phases with critical value $\rho_{c}=0.5$ that correspond to the maximal value. Based on the case of Boltzmann entropy, the arrangement of the binary sequence can be organized or random. For example if $N=L / 2$, as there are $C_{L}^{N}$ microstates of placing the values $(1,0)$ on lattice of length $L$, some microstates can be described, from the perspective of randomness, as well organized, for example the two microstates defined by the relations $x=\left[1_{1 \times L / 2}, 0_{1 \times L / 2}\right]$ and $x=\left[0_{1 \times L / 2}, 1_{1 \times L / 2}\right]$, where $L$ is supposed to be even number, are not random configurations. Another example is the case of periodic sequence such that if $i$ is even then $x_{i}=1$ and if $i$ is odd then $x_{i}=0$ for $i=1, \ldots, L$, this deterministic sequence is not random, and other microstates are characterized by randomness, thus a quantification of the randomness of the binary sequence may require additional metrics and is subject to ongoing researches. As second part, we present in the next section a metric in order to measure the randomness characteristic.

\section{Randomness of Binary Sequences}

The principal parameters of the binary sequence $x$ are the length $L$ and the density $\rho$, given any value of the density, the corresponding number of microstates is $\Omega=C_{L}^{N}$, the repartition characteristic of $x$ for the values 1 and 0 requires a metric to quantify the degree of randomness. Among the standard methods of randomness characterization are the analysis of the entropy functions, the power spectral density and the correlation function. A recently proposed metric [17] is based on diffraction theory, where the output expression of the electric field from the diffraction grating in far field zone from the diffraction plan depends on the number and the geometrical characteristics of the apertures. The metric is based on the hypothesis of periodic and uniform diffraction grating where the apertures are identical and have infinitesimal width. The expression of the function is given as follows:

$$
A=\frac{1}{\sqrt{M}} \sum_{n=1}^{L} x_{n} e^{j 2 \pi n / L}
$$

Where $j=\sqrt{-1}$ and $M=\sum_{n=1}^{L} x_{n}$, the expression of this function is also based on the quantity metric for measuring the localization properties of the wavefunction in the field of condensed matter physics [17]. The variable $x_{n}$ corresponds to the probability amplitude of the electron at the $n^{\text {th }}$ site of lattice that consists of $L$ sites. The performance analysis of the function $A$ was based on the effect of the length of the sequence $x$, and comparatively to the Lyapunov exponent of the logistic map [5,17] defined by $x_{n+1}=\mu x_{n}\left(1-x_{n}\right)$ such that the sequence was transformed into binary values using a threshold $d=0.5$, the comparison of the variation of $A$ and Lyapunov exponent $\lambda$ demonstrated their consistency with respect to the growth parameter $\mu$. Similarly, we introduce a metric in order to characterize the randomness of $x$ based on the theory of the antennas and propagation, precisely the equation of the array factor.

The analysis of the arrays of antennas [19] plays crucial role in several scientific fields including telecommunications [25], acoustics and radars. The geometrical configuration and the properties of the antennas have an impact on the radiation characteristics of the electric or acoustic field. The standard model of the array of antennas is uniform linear array where all the elements are identical and equally spaced. The electronic adjustment 
and digital signal analysis of the antennas allow to study the properties of the propagating electric field such as steering the radiation towards receivers, the objectives are the reduction of the electromagnetic pollution in the environment and saving the electromagnetic energy. These objectives are realizable using high resolution methods [19] for estimating the properties of the radiating sources including theirs powers and angles of incidence. Among the important properties of the uniform linear array is the radiation pattern which is result of superposition of the fields of each element as an interference pattern. For simplicity we briefly present the array factor for uniform linear array of isotropic antennas. We consider that in $(x, y)$ plan, $L$ dipoles parallel to the $z$ axis are placed along $x$ axis with same inter-element distance $d$, each element is characterized by gain $g_{n}(\theta)=1$ for any value of the angle $\theta \in[0, \pi]$. Each element is described by its complex current $I_{n}$ which represents the input amplitude and phase. Given the length of the array $D=(L-1) d$, in the far field zone $r$ described by the criterion $r \geq 2 D^{2} / \lambda$ where $\lambda$ is the wavelength, the array factor [19] $A F(\theta)$ is defined by the following equation:

$$
A F(\theta)=\sum_{n=1}^{L} I_{n} e^{-j k d \cos (\theta)(n-1)}
$$

$k=2 \pi / \lambda$ is the wavenumber and $\theta$ is the azimuth angle. The variations of the defined parameters $I_{n}, \lambda, L$ and $d$ induce a change in the interference pattern of $A F(\theta)$. Based on the expression of the array factor, we introduce a variant of the metric $A$ in order to characterize the randomness of the binary sequence. For this purpose we make a correspondence between the parameters of each quantity. Firstly, the complex current of the $n^{\text {th }}$ antenna $I_{n}$ corresponds to the $n^{\text {th }}$ variable $x_{n}$, secondly we consider the particular value of the azimuth angle $\theta=0^{\circ}$. Next, we define the remaining quantity by $k d=2 \pi / L$. Using the factor $1 / \sqrt{L}$, we obtain a characterization metric of $x$ denoted by letter $\Psi$ and defined by the expression:

$$
\Psi=\frac{1}{\sqrt{L}} \sum_{n=1}^{L} x_{n} e^{-j 2 \pi(n-1) / L}
$$

Note that a second notation can also be obtained using the average operator, given a linearly increasing vector $l=[0,1, \ldots, L-1]$, the second expression of this operator is given by:

$$
\Psi=\sqrt{L}<x e^{-j 2 \pi l / L}>
$$

By comparing the expressions of $\Psi$ and $A$, we remark that they share a common property which consists of the sum of the elements of the sequence $x$ where each element $x_{n}$ is multiplied by an exponential factor that depends on the position $n$. However the proportionality factor and the expression of the exponential factor are different, thus it is required to compare the performance of both operators based on the variations of the different parameters of the sequence $x$. We consider first, a comparison based on logical table where the input sequence is two dimensional $x=\left[x_{1}, x_{2}\right]$, as we have $\Omega=2^{2}$ microstates, we compute the response of $\Psi,|A|$, the information entropy $h$ and the exclusive OR operator known as XOR operator 
given by the equation $\operatorname{XOR}\left(x_{1}, x_{2}\right)=\bar{x}_{1} x_{2}+x_{1} \bar{x}_{2}$, the obtained results are presented in Table 1.

Table 1. Comparison of the Output Values of the Different Metrics with Respect to the Microstates of Binary Vector $x=\left[x_{1}, x_{2}\right]$

\begin{tabular}{ccccc}
\hline Microstate & $h$ & $\Psi$ & $|A|$ & $X O R$ \\
\hline$x=\left[\begin{array}{ll}0 & 0\end{array}\right]$ & 0 & 0 & $\#$ & 0 \\
\hline$x=\left[\begin{array}{ll}1 & 0\end{array}\right]$ & $\log (2)$ & $1 / \sqrt{2}$ & 1 & 1 \\
\hline$x=\left[\begin{array}{ll}0 & 1\end{array}\right]$ & $\log (2)$ & $1 / \sqrt{2}$ & 1 & 1 \\
\hline$x=\left[\begin{array}{ll}1 & 1\end{array}\right]$ & 0 & 0 & 0 & 0 \\
\hline
\end{tabular}

The variations of the functions $\Psi, h$ and XOR are consistent given the probability coefficients $p=[p(0), p(1)]$ for the microstates which are given respectively by $p=[1,0], p=[0.5,0.5], p=[0.5,0.5]$ and $p=[0,1]$. Since the operator $A$ is inversely proportional to the square root of the sum of $x$, the output for the microstate $x=[0,0]$ is not defined. We consider a sequence with longer length $L>>1$.We compute the theoretical expressions for some standard microstates of $x$, for the first microstates of zeros, we can easily verify that $\Psi\left(x=0_{1 \times L}\right)=0$, for the complementary case, we obtain the same result as follows:

$\Psi\left(x=1_{1 \times L}\right)=\frac{1}{\sqrt{L}} \sum_{n=1}^{L} e^{-j 2 \pi(n-1) / L}=\frac{1}{\sqrt{L}} \sum_{n=0}^{L-1} z^{n}=\frac{1}{\sqrt{L}} \frac{1-z^{L}}{1-z}=0$

Where $z=e^{-j 2 \pi / L}$, thus for binary sequence $x$ with varying density $\rho=\langle x\rangle$, the boundary values of $\Psi$ are equal to zero. For critical density $\rho_{c}=0.5$, we consider a simple microstate given by $x=\left[1_{1 \times L / 2}, 0_{1 \times L / 2}\right]$, the value of the operator is equal to:

$$
|\Psi|=\left|\frac{1}{\sqrt{L}} \sum_{n=1}^{L / 2} e^{-j 2 \pi(n-1) / L}\right|=\sqrt{\frac{2}{L\left(1-\cos \left(\frac{2 \pi}{L}\right)\right)}}
$$

Comparatively we calculate the corresponding value for the metric $A$ such that $M=L / 2$, the theoretical expression is given by:

$$
|A|=\left|\sqrt{\frac{2}{L}} \sum_{n=1}^{L / 2} e^{j 2 \pi n / L}\right|=\sqrt{\frac{4}{L\left(1-\cos \left(\frac{2 \pi}{L}\right)\right)}}
$$

For given microstate other than the cited above, it may be difficult to derive the theoretical expression, therefore it is necessary to conduct the numerical tests to compare the behaviors and the fluctuations of $A$ and $\Psi$ with respect to the different parameters. In the next section, we present and discuss some numerical results.

\section{Computational Results}

In this section, we present the conducted numerical tests to study the variations of the proposed metric $\Psi, A$ and Shannon entropy $h$ by varying the different parameters of $x$. 
For the first simulation test, we consider a binary sequence with length $L=200$, the value 1 is affected to variable $x_{n}$ if random number in the range $[0,1]$ is less than the density $\rho$. By varying the density starting from the lower value $\rho=10^{-4}$ since $A$ is not defined for $\rho=0$, and incrementing the density with rate $d \rho=0.01$, we compute for each value the functions $\Psi$ and $A$, each result of the operators is averaged over $T=200$ trials. The obtained functions are presented in Figure 1 .

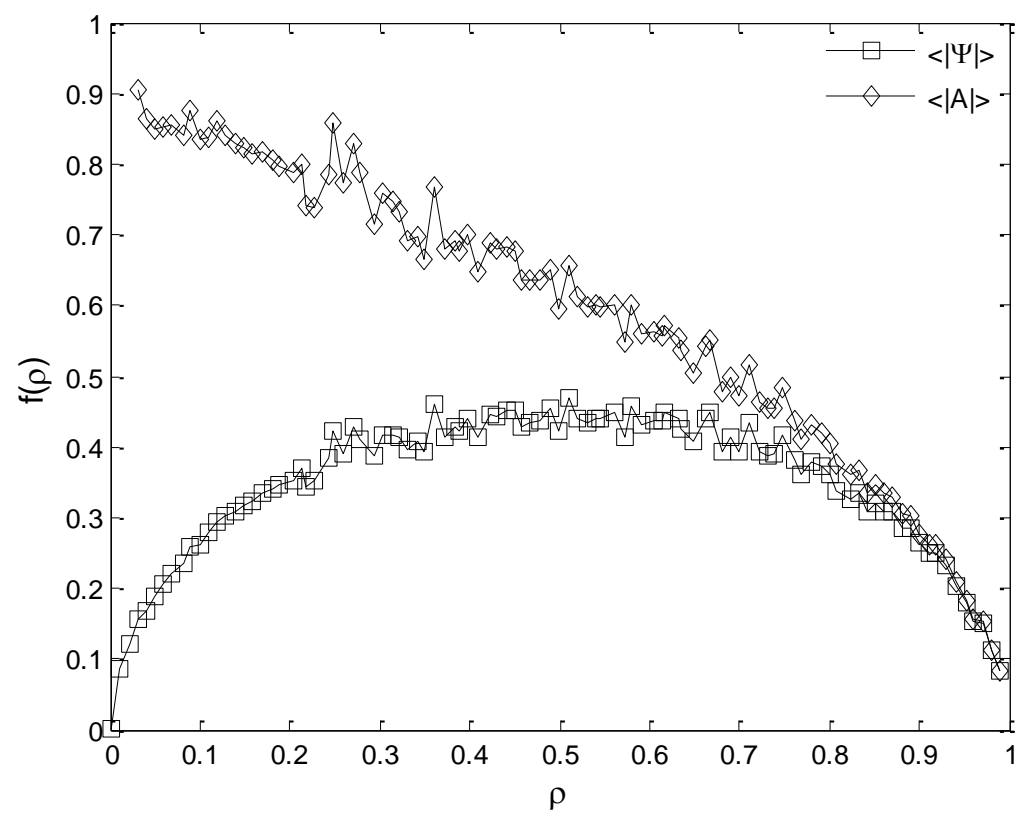

Figure 1. Variations of the Operators $\Psi$ and $A$ with Respect to the Density $\rho$ for Binary Sequence $x$ with $L=200$

The metric $A$ decreases non linearly with increasing density and equals zero for $\rho=1$, the randomness characteristic is reflected for lower values of the density, the interpretation of the response $A$ indicates that the randomness decreases with increasing density, note that $A$ is not defined for $\rho=0$ as the simulation started with value $\rho=10^{-4}$. In the other hand, the variation of $\Psi$ is consistent with that of Shannon entropy $h$ such that $\Psi$ is maximal at the critical density $\rho=0.5$ and symmetric with respect to this value. As these results are the average values, we examine the fluctuations of the operators as a function of the number of the trials. For the second test, we fix the value of the density at $\rho=0.5$ and we consider the number of trials $T=400$, for each trial a new sequence $x$ is generated and the metrics are computed, the obtained results are illustrated in Figure 2. 


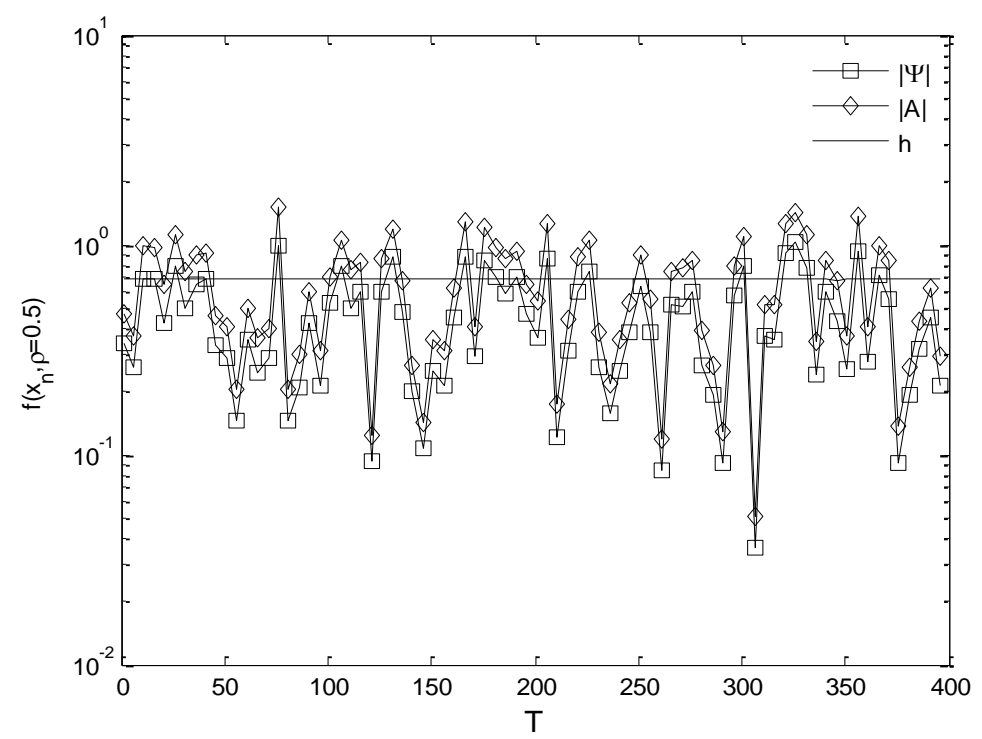

Figure 2. Variations of the Operators $\Psi, A$ and $h$ for Binary Sequence $x$ with Length $L=200, \rho=0.5$ and $T=400$

We remark that both $\Psi$ and $A$ fluctuate with approximately the same amplitude while $h$ is constant since for each trial, the density $\rho$ is fixed such that $h$ does not depend on the geometric repartition of the binary values. This result implies that for varying $\rho$, the metric $\Psi$ is consistent with $h$, and for critical density $\rho=0.5$ the variations of $\Psi$ and $A$ are approximately equivalent. We exploit the symmetrical property of $\Psi$ and we evaluate its variation as function of the length $L$, we consider different values of the density $\rho=0.1, \rho=0.2$ and $\rho=0.5$, we vary the parameter $L$ such that for each value an average of $\Psi$ over $T=100$ trials is computed. The results are presented in Figure 3.

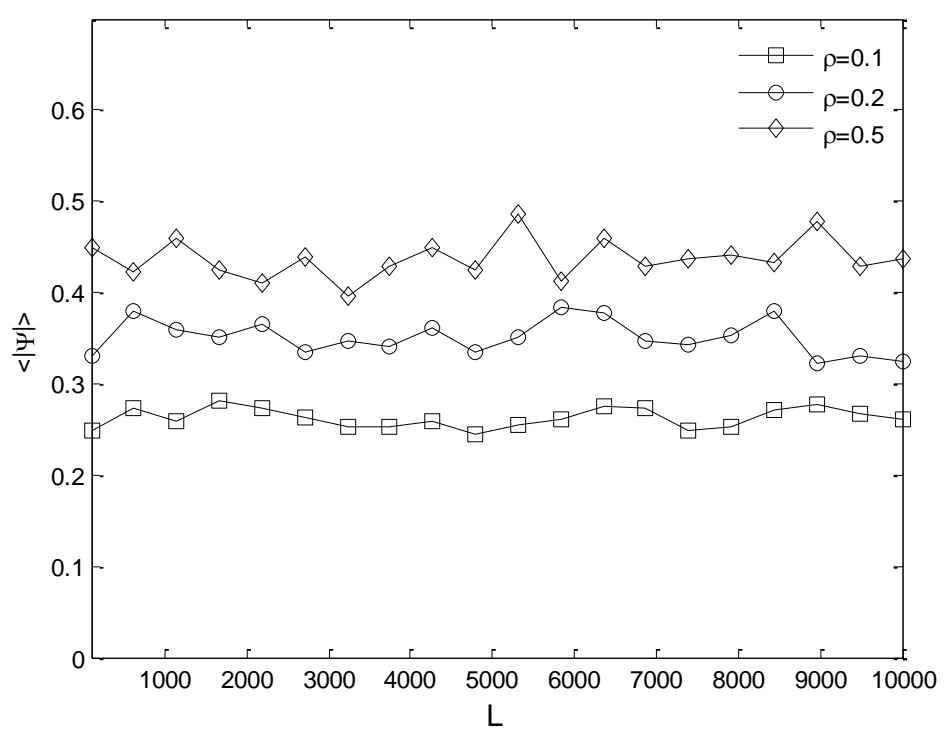

Figure 3. Average Variation of $\Psi$ as Function of Length $L$, for Different Values of $\rho$ 
As presented in Figure 1, $\Psi$ increases non linearly with increasging density in the range $[0,0.5]$. By varying the length $L$ in the result of figure.3, the order of this variation is preserved as we have $\Psi(\rho=0.5, L)>\Psi(\rho=0.2, L)>\Psi(\rho=0.1, L)$.

The random binary sequence can be generated from the logistic map as it was explained in [17], it is model of non linear dynamical system defined by the iterative equation $x_{n+1}=\mu x_{n}\left(1-x_{n}\right)$ defined by the growth parameter $\mu \in[0,4]$ where the range of the sequence is $x_{n} \in[0,1]$. The binary sequence of symbols was generated using a transformation based on threshold $d=0.5$ and the metric $A$ was applied comparatively to the Lyapunov exponent [5,17] which is an indicator of the chaotic behavior of the sequence $x_{n}$ with respect to the parameter $\mu$. In our case, we consider a binary sequence that is derived based on logarithmic logistic map defined by $y_{n}=-\mu y_{n} \log \left(y_{n}\right)$ which is nonlinear dynamical model proposed to study the stability of the Greenberg's model of traffic flow with normalized jam density. The range of the parameter that represents the optimal velocity is $\mu \in[0, e]$ and also the range of the sequence is $y_{n} \in[0,1]$. The chaotic phase is defined by the interval $[2.5, e]$. Using the following transformation:

$x_{n}=\left\{\begin{array}{lll}0 & \text { if } & y_{n}<d \\ 1 & \text { if } & y_{n}>d\end{array}\right.$

Where $d=0.5$, we obtain the binary sequence $x_{n}$ that depends on $\mu$, therefore we evaluate the operator $\Psi$ comparatively to $A$ by varying $\mu$. We consider that the initial length of the sequence is $N=10^{4}$ and the initial condition is random variable $y_{1} \in[0,1]$. For each value of $\mu$ varying with rate $d \mu=0.005$, we generate $y_{n}$ and consider the last $L=2000$ values to transform $y_{n}$ into binary sequence $x_{n}$. For each value of $\mu$ the normalized metrics are computed. We present the obtained results in Figure 4.

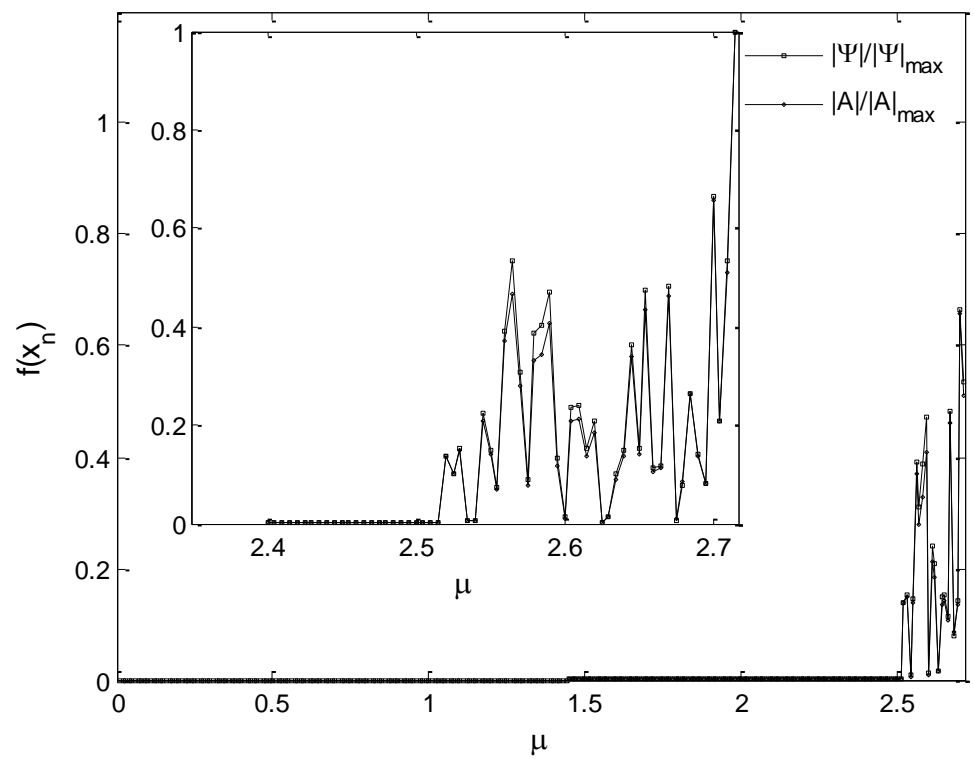

Figure 4. Variations of the Metrics $\Psi$ and $A$ as Function of $\mu$ for Binary Sequence Obtained from Logarithmic Logistic Map, $L=2000$ 
The metric $\Psi$ equals zero in the range $[0,2.5]$. In the chaotic phase, the variations of both functions are approximately equivalent, the variations in the chaotic region are illustrated in the zoomed part of the figure, we remark that the normalized metrics $|\Psi| /|\Psi|_{\max }$ and $|A| /|A|_{\max }$ vary almost with the same rate of the fluctuations, which implies the randomness characteristic of binary sequence $x_{n}$. The Lyapunov exponent of the logarithmic logistic map $\lambda(\mu)$ reflects the chaotic behavior of the sequence $y_{n}$, it is given by:

$$
\lambda(\mu)=\frac{1}{N} \sum_{n=1}^{N} \log \left|\left(\frac{d f(y)}{d y}\right)_{y_{n}}\right|=\frac{1}{N} \sum_{n=1}^{N} \log \left(\left|-\mu\left(\log \left(y_{n}\right)+1\right)\right|\right)
$$

In [17], the authors compared the variation of the corresponding Lyapunov exponent of the standard logistic map with the variation of the operator $A$ by varying the growth parameter $\mu$. Similarly, based on logarithmic logistic map, we compare the variations of $\Psi$ and $A$, applied on binary sequence $x_{n}$, against the Lyapunov exponent, the results are presented in figure.5, using the normalization for better comparison and using initial length $N=10^{4}$ and length of the sequence $L=8000$.
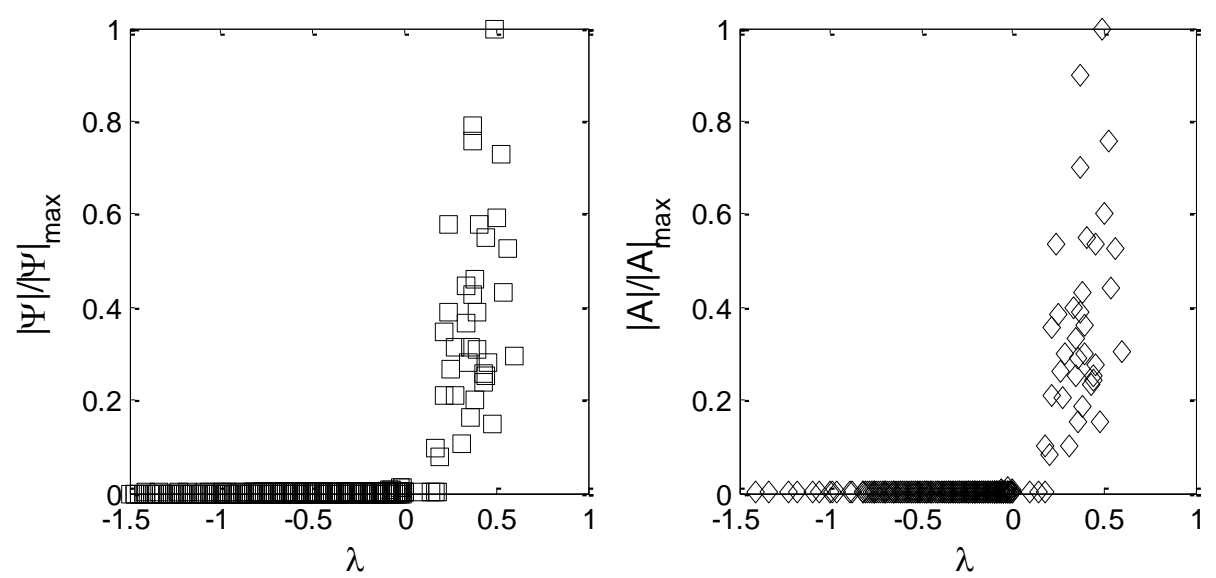

\section{Figure 5. Variations of the Metrics $\Psi$ and $A$ for Binary Sequence $x_{n}$ against the Corresponding Lyapunov Exponent $\lambda(\mu)$ of the Initial Sequence $y_{n}$}

For the negative values of the Lyapunov exponent, the values of $\Psi$ and $A$ equal zero, and approximately positive values of $\lambda(\mu)$ correspond to positive values of both metrics, neglecting the values of the amplitude, we remark from the figure that their variations are almost the same reflecting the chaotic behavior of the binary sequence $x_{n}$. We conclude from these simulation results that the operator $\Psi$ can be applied to study the parameters of the binary sequence from probabilistic approach since it is consistent with the information entropy, and from the perspective of the randomness characteristic of the binary sequence $x_{n}$.

As perspective, it is logical to search for some common aspects between $\Psi$ and the Fourier transform of binary sequence $x_{n}$, and also to change the proposed parameters of $\Psi$ and test for the corresponding results using different parameters 
including $\rho$ and $L$. A second possible research consists of studying the variation of $\Psi$ as function of several binary sequences.

\section{Conclusion}

We have presented, in this paper, a study of binary sequences defined by the values $(0,1)$. In the first part, the statistical parameters were derived using the average value of sequence, including the standard deviation, the variance coefficient, the skewness and the kurtosis. In the second part, several entropy functions were also reviewed where their expressions were simplified, including Rény entropy with parameter $\mathrm{q}=2$, Tsallis and Boltzmann entropies. In the second part, we partially treated the randomness characteristic of the binary sequence using the density, the length and the method for generating random binary sequence, we have presented a scalar function based on the equation of the array factor which is used in the field of antennas and propagation, numerical evaluations were performed comparatively to a recently proposed function inspired from the diffraction theory. The simulation results indicated the consistency of the proposed function with the different entropy functions such as Shannon entropy. For binary sequence generated from logarithmic logistic map, the variation of the presented metric and the diffraction based function were approximately equivalent.

\section{References}

[1] G. F. M. Beenker, T. A. C. M. Claasen and P. W. C. Hermens, "Binary sequences with a maximally flat amplitude spectrum", Philips J. Res., vol. 40, (1985), pp. 289-304.

[2] R. Turyn and J. Storer, "On binary sequences", Proc. Am. Math. Soc., vol. 12, (1961), pp. 394-9.

[3] S. S. Melnik and O. V. Usatenko, "Entropy of finite random binary sequences with weak long-range correlations", Phys. Rev. E 90, 052106, (2014).

[4] F. Akhter and M. S. A. Mamun, "Pseudo random binary sequence: A new approach over finite field and its properties", 2017 International Conference on Electrical, Computer and Communication Engineering (ECCE), Cox's Bazar, (2017), pp. 676-680.

[5] M. J. Páez, C. C. Bordeianu and R. H. Landau, "A Survey of Computational Physics Introductory Computational Science", (Princeton University Press, 2008), pp. 688.

[6] S. Sajic, N. Maletic, B. M. Todorovic and M. Sunjevaric, "Random Binary Sequences in Telecommunications", Journal of Electrical Engineering, vol. 64, no. 4, (2013), pp. 230-237.

[7] M. J. E. Golay, "A class of finite binary sequences with alternate autocorrelation values equal to zero", IEEE Trans. Inf. Theory, (1972).

[8] D. Torrieri, "Principles of Spread-Spectrum Communication Systems", Springer, (2005).

[9] I. A. Pasha, P. S. Moharir and N. S. Rao, "Bi-alphabetic pulse compression radar signal design Sãdhanã", vol. 25, pp. 481-8.

[10] E. Maciá, “Aperiodic Structures in Condensed Matter: Fundamentals and Applications”, CRC Press, Boca Raton, (2009)

[11] R. Durbin, S. Eddy, A. Krogh and G. Mitchison, "Biological Sequence Analysis", Cambridge University Press, (1998).

[12] D. Lind and B. Marcus, "Symbolic Dynamics and Coding", Cambridge University Press, (1995).

[13] K. Nagel and M. Schreckenberg, J. Phys. I France 2, 2221, (1992).

[14] J. Esser and M. Schreckenberg, "Microscopic Simulation of Urban Traffic Based on Cellular Automata", International Journal of Modern Physics C 1997 08:05, pp. 1025-1036.

[15] T. Packebusch and S. Mertens, "Low autocorrelation binary sequences", J. Phys. A: Math. Theor. 49 (2016) 165001 (18pp).

[16] O. V. Usatenko, S. S. Melnik and S. S. Apostolov, "Iterative Method for Generating Correlated Binary Sequences", arXiv:1406.2656v1, (2014).

[17] L. Gong, H. Wang, W. Cheng and S. Zhao, "A measurement of disorder in binary sequences", Physica A 422, (2015), pp. 66-72.

[18] O. V. Usatenko, S. S. Melnik, L. Kroon, M. Johansson, R. Riklund and S. S. Apostolov, Physica A, 387 (2008) 4733

[19] F. Gross, "Smart Antennas for Wireless Communications", McGraw-Hill Professional, (2005) September.

[20] Z. Yu-mei and Q. Shi-ru, "Research on Chaotic Characteristics for Freeway Traffic Flow", 2009 International Conference on Measuring Technology and Mechatronics Automation. 
[21] C. E. Shannon, “A mathematical theory of communication”, Bell System Technical Journal, vol. 27, no. 3, (1948) July, pp. 379, 423.

[22] J. Zhang, Y. Zhang and C. Yu, "Rényi entropy uncertainty relation for successive projective measurements", Quantum Inf Process, (2015), 14:2239.

[23] W. Tatsuaki, "On the thermodynamic stability conditions of Tsallis' entropy", arXiv:condmat/0201368v2, (2002).

[24] C. Tsallis, F. Baldovin, R. Cerbino and P. Pierobon, "Introduction to Nonextensive Statistical Mechanics and Thermodynamics", arXiv:cond-mat/0309093v1, (2003).

[25] Y. Khmou, S. Safi and M. Frikel, "Lorentzian Operator for Angular Source Localization with Large Array", Journal of Telecommunications and Information Technology, vol. 4, (2015), pp. 98-105. 